\title{
Percentage of pupillary dilation as a measure of item difficulty'
}

\author{
DONALD T. PAYNE, ${ }^{2}$ MARY ELLEN PARRY AND STEFAN J. HARASYMIW \\ EDUCATIONAL TESTING SER VICE
}

The purpose of this study was to compare four measures of difficulty of mental multiplication items: percentage of pupillary dilation, latency of solution, number of correct responses, and judgment of item difficulty. Sixteen multiplication problems, classified into four levels of difficulty, were presented visually to $13 \mathrm{Ss}$, who verbalized their solutions to the problems. Analyses of variance and correlation coefficients were computed. It was concluded that all four measures of difficulty were useful but that judgment of difficulty and latency of solution were better measures of item difficulty than were the other two. A discussion of pupillary dilation and information processing is included.

Within the past three years more than a dozen reports have appeared that have presented evidence of relationships between measures of dilation of the pupil and hypothetical intellective or affective processes that were assumed to have been elicited by the stimulus materials administered in the experiments. One purpose of the present study was to compare a measure of the pupillary response with other measures of item difficulty: latency, correctness of response, and judgment of difficulty. The second purpose was to determine whether results similar to those reported by Hess and Polt (1964) would be obtained under different experimental conditions.

Hess and Polt reported a direct relationship between the difficulty of a mental multiplication task and pupillary dilation. In their experiment, a series of four multiplication items, presented in order of difficulty, was read (e.g., "multiply eight times thirteen") to five Ss who reported their answers orally. The average percentage of dilation over Ss increased with each successive item.

Several changes in experimental conditions were made in the present study, such as visual instead of oral presentation of multiplication items and the almost complete removal of the visible component of the illumination used for photographing. A major refinement was the use of an operational definition of item difficulty in specifying the population of items sampled in the study.

\section{Test Items}

\section{METHOD}

A multiplication algorithm was identified and item difficulty was defined according to processing differences resulting from application of the algorithm to specific items. Subsequently a simpler but coordinate definition in terms of the physical properties of items was discovered. Since the description of the algorithmic definition is lengthy, the simpler definition is presented. By this definition the population of items and levels of difficulty were specified as follows:

(1) Only items with single-digit multipliers were considered.

(2) Four levels of difficulty were identified. From easier to more difficult they consisted of items that had multiplicands of from one to four digits.

(3) Items within levels were selected to be of similar difficulty. The population of items at each level was defined so that solution protocols of identical kinds and numbers of operations resulted when the multiplication algorithm was applied. These conditions were satisfied when the range of digit values was restricted to the values 4 through 9.3 To reduce item variability even further digit values from 4 through 7 were used.

From this definition four problems at each of the four levels of difficulty were generated using a table of random numbers. (Table 2 , presented in the Results section, enumerates the items used.) Sixteen test stimuli were prepared as slides. A control slide, similar to that used by Hess and Polt, displayed one of the numbers from one to four in each corner and the number five in the center of the slide.

\section{Subjects}

The 13 Ss were all females and were high school, but not college, graduates. They ranged in age from 18 to 25: an upper limit of age 25 was employed to reduce the effect of accommodation (Woodmansee, 1966). Within these limits Ss constituted an incidental sample of convenient volunteers.

\section{Apparatus}

Stimuli were rear-projected on a $9 \times 12$ in. Polacoat screen mounted on a vertical panel $36 \mathrm{in}$. in front of S. The control slide and test slides were mounted in two different projectors to reduce greatly the effect of darkness during the slide changing interval. Both projectors were $500 \mathrm{~W}$ units with adjustable diaphragms (set at about .25 in. diameter) mounted on their lenses to reduce the light intensity and to allow matching the intensity of the reflected light from both projectors.

$S$ sat on a chair that was adjustable in height and rested her forehead on a fitted headrest, but was otherwise not physically restrained. S's right eye was photographed twice every second by a single-frame $16 \mathrm{~mm}$ camera centered immediately below the screen. A large image of the pupil was obtained at the film plane by using a $200 \mathrm{~mm}$ lens and extension tubes.

Kodak Hi-Speed Infra-red film was used in the camera. The right eye was illuminated by a third slide projector fitted with a Kodak 87 filter, which passed infra-red but removed nearly all visible light. Solid state logic circuitry was used to control all pieces of apparatus, including an electro-magnetic latency counter and the camera.

\section{Procedure}

The order of presentation of the four slides within each of the four levels was systematically changed for each $S$. Of the possible 24 orders of four, 13 different orderings were used. For all Ss, however, problems from the easiest level of difficulty were presented first, with each successive level becoming more difficult. The experiment was conducted in a room with very low general illumination. Noise from the fans of the three projectors tended to mask transient noise such as that emitted by the slide changing mechanism.

Each S was instructed to solve multiplication problems in her head and give the answer orally as soon as she reached the solution. Ss were told to use the traditional method of multiplication and to try for both speed and accuracy. $S$ was not told if her answer was right or wrong. When the control slide appeared between each problem slide $S$ was requested to look at each of the five numbers on the slide in succession. $S$ was asked not to move her head or close her eyes during the experiment. One practice problem was presented and solved by $\mathrm{S}$. The camera was then turned on, and the control slide presented.

The experiment proper began with the presentation of the first test problem. As soon as $S$ completed verbalizing her solution, $E$ stopped the latency counter which caused the control slide to supplant the test item on the screen. The control slide remained on for a fixed interval of $8 \mathrm{sec}$. The cycle was then repeated until all 16 test items had been presented.

$S$ was then seated at another table and given instructions for the next task. The 16 multiplication problems that had just been solved were presented again; each item was individually typed on a $3 \times 4$ in. sheet of paper. $S$ was asked to judge the difficulty of the 
items by assigning number 1 (to the easiest) through 20 (to the most difficult) according to her judgments of relative difficulty. The same number was to be assigned to more than one problem if she felt that two or more problems were of equal difficulty.

\section{Measurements}

Four different response measures were collected from the administration of the multiplication items: (1) Latency-the interval from item presentation to the time immediately after $S$ completed verbalizing the item solution was measured in tenths of seconds, (2) Correctness of Response, (3) Judgment of Difficultythe number (1-20) that was assigned to each item by $S$ in the second part of the experiment, and (4) Pupil Size-the horizontal diameter of the image of the pupil was measured on each frame of film. The measurement was the count of the number of cross-sections of a graph subtended by a projected image of the pupil. These diameters were all measured by one person who was not involved in the data collection. In several places in this report the pupillary diameter as measured from the projected image is reported. An approximate value for actual pupillary diameter can be obtained by multiplying these figures by 0.086 .

The reliability of measuring the diameter of the pupil was checked as follows: the first 68 frames and the last 92 frames of the responses of one $S$, chosen at random, were remeasured after all measurements were completed. All frames classified as blinks on either or both readings were considered as blinks and not included in the reliability computation. The Pearson productmoment correlation among the remaining 64 pairs of readings was .96 and among the remaining 83 pairs was .92 .

Hess and Polt computed percentage of dilation from the difference in average pupil diameter between a $2.5 \mathrm{sec}$ segment of the control and subsequent problem solving interval. A more conservative measurement was made in this study by averaging all pupil diameters measured during the respective intervals. The percentage of pupillary dilation was computed by taking the difference between the average diameter during the problem interval and the average of the control intervals preceding and following the problem interval, dividing it by the average of the two control intervals, and multiplying by 100 .

\section{RESULTS}

An analysis of variance was computed for each of the dependent variables (excluding correctness of solution) collected in this study. The experimental design of the study was a partial hierarchical design with repeated measures over two factors and with one factor (Items) necessarily nested within the other (Levels of Difficulty). The third factor was Subjects. The Subjects and Items factors were considered as random and the Levels of Difficulty factor as fixed.

The analysis of variance results for each dependent variable are presented in Table 1.4 The significance of each effect listed in the table, except Levels of Difficulty, are determined by computing an $F$ ratio between that effect and the Residual term. Since the model for the analysis did not provide an appropriate $F$ ratio for testing the significance of the Levels of Difficulty factor, quasi $F$ ratios were computed (Winer, 1962). Every $F$ ratio is significant beyond the .01 level.

The Subjects by Levels of Difficulty interaction is explained by individual differences in the amount of the increase in average responses to items within a level from level to level. In no case did an S's average latency or judgment response value decrease in moving from a lower level to the next higher level of difficulty. The individual average of percentage of pupillary dilation did vary both in amount and direction of change from level to level.

The fact that there was a significant amount of variability among items within levels indicates a lack of item homogeneity. An obvious attribute to suspect is digit size. To check this - hypothesis each item was assigned a digit size score equal to the sum of the values of each digit in the item. These scores and both the mean latency and judgment measures for each item were converted to ranks and Spearman rank correlation coefficients were computed between the digit size and both the other
Table I

Analysis of Variance Summary Table

\begin{tabular}{lrccc} 
& \multicolumn{4}{c}{ F Ratio $^{\mathrm{a}}$} \\
\cline { 2 - 5 } \multicolumn{1}{c}{ Source } & \multicolumn{1}{c}{$\begin{array}{c}\text { Percentage of } \\
\text { Pupillary Dilation }\end{array}$} & $\begin{array}{c}\text { Latency } \\
\text { of Solution }\end{array}$ & $\begin{array}{c}\text { Judgment of } \\
\text { Item Difficulty }\end{array}$ \\
\hline Subjects & 12 & 30.386 & 5.700 & 8.225 \\
Levels of Difficulty & 3 & $12.627^{\mathrm{b}}$ & $17.736^{\mathrm{c}}$ & $102.004^{\mathrm{d}}$ \\
Items within Levels & 12 & 3.316 & 3.118 & 9.482 \\
Subjects x Levels & 36 & 8.886 & 2.220 & 2.655 \\
Residual & 144 & & & \\
\hline
\end{tabular}

$a_{A}$ All ratios are significant at the .01 level.

${ }^{b}$ Quasi $F$ ratio: $F^{\prime \prime}=\left(M S_{L}+M S_{R}\right) /\left(M S_{I w L}+M S_{S x L}\right) ; d f=3 / 48$.

cQuasi F ratio: $d f=3 / 30$

$d_{\text {Quasi }}$ F ratio: $d f=3 / 19$

measures. Over all 16 items the correlations were equal to or greater than 0.98. Six of the eight correlations within levels were equal to or greater than .95 , the remaining two being 0.79 and 0.63 . This evidence strongly supports the hypothesis that item variability within levels is due to the variability of digit values among items.

One comparison among all four of the dependent variables can be made by examining the mean responses to each item as they are presented in Table 2. The items are ordered in the table according to the value of the mean latency. Except for one inversion (item numbers 10 and 12), items are ordered alike on both latency and judgment of difficulty for Levels 2,3 , and 4 . The ranking of mean number of correct responses agrees with latency rankings only within Level 3. Rankings of per cent dilation correspond with rankings of latency only on items within Level 1.

Several other interesting facts can be observed from Table 2. All $13 \mathrm{Ss}$ agreed in assigning a 1 (the lowest value) to item number 1 and a 20 (the highest value) to item number 14. The measure of pupil dilation appears to discriminate poorly among the more difficult items while the mean number of correct responses is very

Table 2

Item Content and Mean Response Values for Each Item for Each Dependent Variable

Mean Values of Dependent Variables

\begin{tabular}{|c|c|c|c|c|c|}
\hline \multirow[b]{2}{*}{ Item No. } & \multicolumn{5}{|c|}{ Mean Values of Dependent Variables } \\
\hline & Item & $\begin{array}{c}\text { Latency } \\
\text { in Seconds }\end{array}$ & Judgment & $\begin{array}{l}\text { Per Cent } \\
\text { Dilation }\end{array}$ & $\begin{array}{c}\text { Proportion of } \\
\text { Correct Solutions }\end{array}$ \\
\hline 1 & $4 \times 5$ & 1.8 & 1.00 & -2.92 & 1.00 \\
\hline 3 & $4 \times 7$ & 2.1 & 2.15 & -1.38 & 0.92 \\
\hline 4 & $5 \times 6$ & 2.2 & 1.38 & 1.19 & 1.00 \\
\hline 2 & $6 \times 7$ & 2.4 & 2.00 & 1.21 & 0.85 \\
\hline Level 1 Means & & 2.1 & 1.63 & -0.48 & 0.94 \\
\hline 7 & $54 \times 5$ & 5.0 & 4.23 & 5.62 & 0.92 \\
\hline 8 & $46 \times 6$ & 5.7 & 5.23 & 5.55 & 0.38 \\
\hline 5 & $74 \times 7$ & 6.5 & 5.92 & 6.27 & 0.85 \\
\hline 6 & $76 \times 6$ & 6.9 & 6.00 & 5.82 & 0.85 \\
\hline Level 2 Means & & 6.0 & 5.35 & 5.81 & 0.75 \\
\hline 12 & $745 \times 5$ & 9.0 & 10.62 & 7.72 & 0.69 \\
\hline 10 & $545 \times 5$ & 9.7 & 9.23 & 6.45 & 0.54 \\
\hline 11 & $675 \times 6$ & 18.1 & 12.00 & 7.61 & 0.23 \\
\hline 9 & $676 \times 7$ & 20.0 & 13.15 & 7.45 & 0.15 \\
\hline Level 3 Means & & 14.2 & 11.25 & 7.31 & 0.40 \\
\hline 16 & $6454 \times 4$ & 20.2 & 16.31 & 6.74 & 0.23 \\
\hline 13 & $4675 \times 6$ & 23.8 & 17.54 & 7.97 & 0.38 \\
\hline 15 & $6474 \times 7$ & 26.3 & 18.85 & 7.71 & 0.15 \\
\hline 14 & $7574 \times 7$ & 31.9 & 20.00 & 8.06 & 0.23 \\
\hline Level 4 Means & & 25.6 & 18.17 & 7.62 & 0.25 \\
\hline
\end{tabular}


similar in value for all the items in Levels 1 and 2 except number 8. No explanation is offered for this inconsistency.

To facilitate comparison among the three dependent variables the percentage of the total variance accounted for by each of the components of the model was computed for each of the variables. As shown in Table 3 comparison of these data shows that Levels of Difficulty accounted for considerably more of the total variance of the judgment variable than is the case for either of the other two variables.

The means of all the variables for each level of item difficulty are plotted in Fig. 1. The scale for each variable was adjusted so that the mean values of all four variables were physically coincident at Levels 1 and 4. Examination of the graph shows that the pattern of means for percentage of pupillary dilation is different from that of the other variables.

To gain information about the consistency of measurements across Ss, intercorrelations between all pairs of the three dependent variables were computed and are presented in Fig. 2. Each point on this figure is the correlation between two measures on one $S$ across all items. The ordering of $S$ s in the figure is according to the value of the latency-per cent pupillary dilation correlation coefficient. This arbitrary ordering was arranged to make the graph easier to follow. Point-biserial intercorrelations were computed between each of the above three variables and correctness of solution and are presented in Fig. 3.

The change in pupillary diameter of a typical $S$ to one item from each level of difficulty is presented in Fig. 4 to illustrate the nature of the pupillary response. The diameter appears to reach a ceiling after a 4 to $5 \mathrm{sec}$ interval.

\section{DISCUSSION AND CONCLUSION}

\section{Comparison of Measures of Item Difficulty}

Each of the four dependent variables collected in this study can be considered as measures of item difficulty. A way of evaluating these measures is to assume that the definition of item difficulty used to generate items of different levels of difficulty is a valid one and to compare differences among the dependent variables in measuring these levels of item difficulty. One comparison is the percentage of the variance of each dependent variable accounted for by the Levels of Difficulty factor. The measure that accounted for the larger portion of the variance is to be preferred. On this basis the three measures (excluding correctness of response) are ranked in order of preference as judgment of difficulty, latency of solution, and percentage of pupillary dilation.

A second comparison is the degree of intersubject variability; the lower the variability the better the measure. The ranking of the three dependent variables from lower to higher intersubject variability is the same as above.

The degree of correlation between two measures of an S's responses to these items is another way of comparing these measures. Latency of solution and judgment of difficulty are consistently highly correlated for all Ss. The correlation between percentage of pupillary dilation and either of the above two measures exhibits a lack of consistency across Ss. Future studies may relate these individual differences in dilation to interesting variables. However, as a measure of item difficulty this variability is undesirable.

The fourth dependent variable was correctness of response. The proportion of correct responses across $S s$ is a traditional measure

Table 3

Percentage of Total Variance Accounted for by Each of the Components in the Model for Each of the Three Dependent Variables

\begin{tabular}{lccc}
\multicolumn{1}{c}{ Source } & $\begin{array}{c}\text { Percentage of } \\
\text { Pupillary Dilation }\end{array}$ & $\begin{array}{c}\text { Latency } \\
\text { of Solution }\end{array}$ & $\begin{array}{c}\text { Judgment of } \\
\text { Item Difficulty }\end{array}$ \\
\hline Subjects & 23.61 & 8.44 & 1.73 \\
Levels of Difficulty & 35.10 & 49.37 & 90.35 \\
Items within Levels & 2.28 & 4.68 & 2.50 \\
Subjects x Levels & 25.98 & 8.77 & 1.59 \\
Residual & 13.04 & 28.73 & 3.83 \\
\hline
\end{tabular}

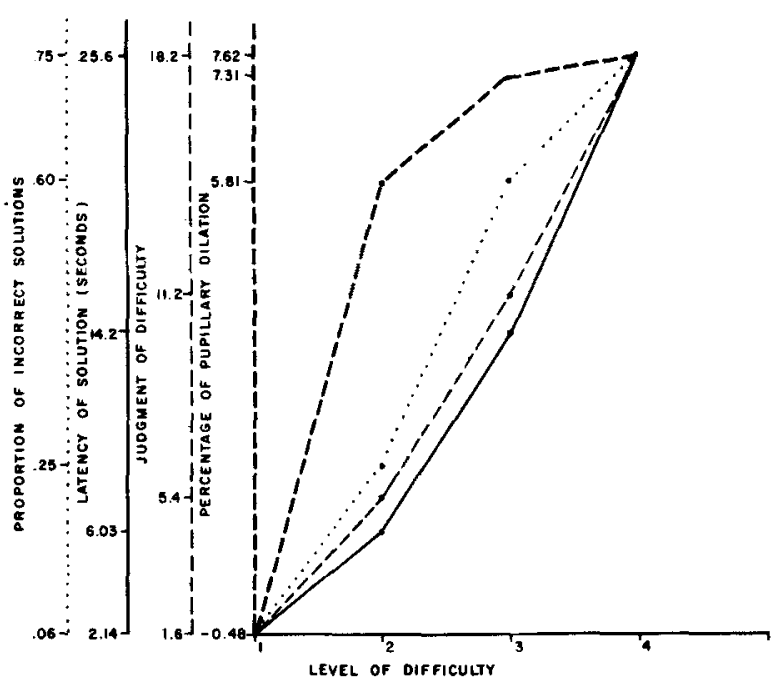

Fig. 1. Mean values at each level of difficulty for each of the four dependent variables.

of item difficulty. This variable was correlated with the other three. Correctness of response correlates highly (negatively) with latency of solution and judgment of difficulty for about half the Ss. The correlation with percentage of pupillary dilation exceeds -.5 for only two Ss.

Finally, it is noted that correctness of response discriminates poorly among the easier items while percentage of pupillary dilation displays the same shortcoming among the more difficult items.

It is concluded that the measures that correspond most closely with the definition of item difficulty are judgment of difficulty and latency of solution. Both percentage of pupillary dilation and correctness of response are deficient in one or more of the comparisons. An additional undesirable feature of the pupillary measure is the difficulty of data collection. However, an advantage of both the pupillary measure and of latency is that a measure of item difficulty is available coincident with solution. There may be need for such instant information in a computer controlled learning environment.

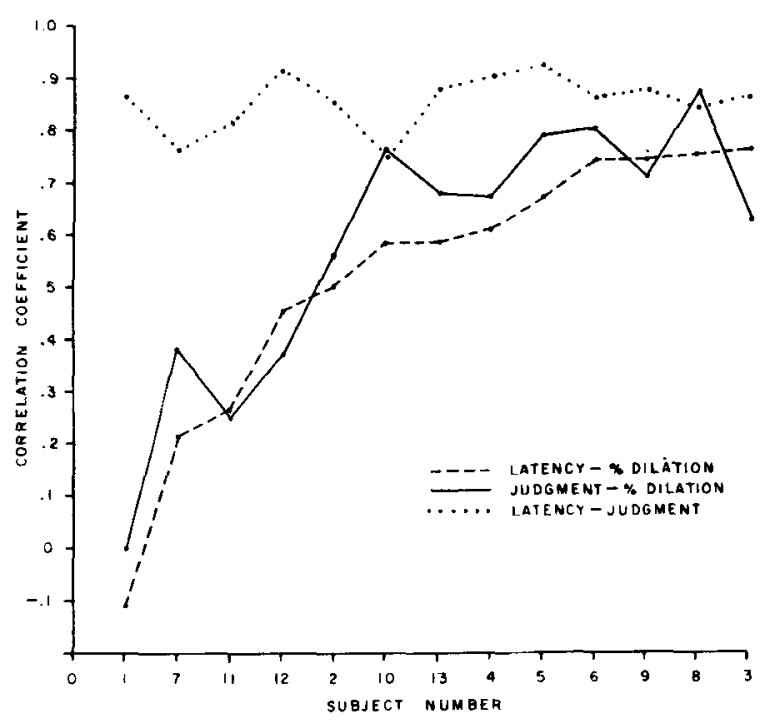

Fig. 2. Intercorrelations between all pairs of the three variables latency, per cent dilation and judgment for each $\mathrm{S}$ across all items. 


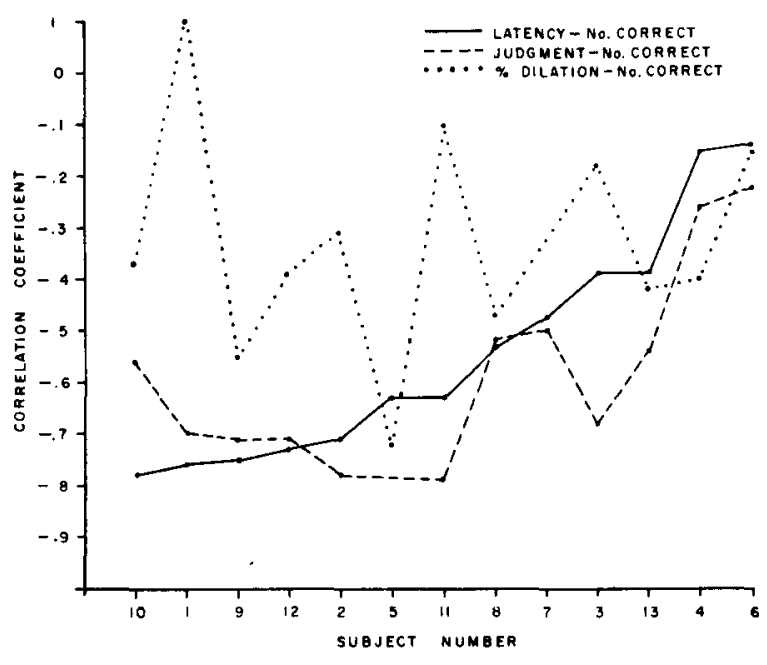

Fig. 3. Intercorrelations between number of correct solutions and each of the other three dependent variables for each $\mathbf{S}$ across all items.

\section{Pupillary Dilation and Information Processing}

The large difference in amount of dilation between Level 1 and all other levels is very interesting when this difference is associated with hypothetical differences in kind of information processing between these same sets of items. It was hypothesized that $S$ obtained the product of two single digit numbers (Level 1 items) by searching a multiplication table in memory and retrieving the product stored there. The solution of all other items required additional processing steps that intuitively seem to be more demanding than that of searching a table of numbers. These results suggest that the extent of dilation may be determined by the kind of information processing in which the $S$ is engaged.

Other results have been reported that are consistent with the above interpretation. Kahneman and Beatty (1966) found that during a short-term memory task (recall of strings of digits) the extent of pupillary diameter varied directly with the length of the string. They described the pupillary response as a measure of the momentary processing load. They also found that a digit transformation task (adding one to the digit) elicited a greater increase in pupillary diameter than did a digit recall task and the peak of dilation for the more difficult task occurred later than it did for the easier one.

In a later study, Kahneman and Beatty (1967) studied pupillary responses in a pitch-discrimination task. Dilation occurred immediately after the presentation of the comparison tone and the magnitude of dilation was directly related to the difficulty of discrimination. These studies support the interpretation that the degree of pupillary dilation increases when the processing task becomes more complex or difficult.

There is another possible explanation for the difference in dilation between Level 1 items and the other items reported in this study. Payne, Parry and Harasymiw (1967) plotted the average pupil diameter of one $S$ over all items at each successive $1 / 2$ sec interval following item presentation. The average response curve accelerated rapidly during the $4 \mathrm{sec}$ following item presentation and then leveled off and remained at a fairly constant value until a solution was reported. The response to each item can be described as a perturbation of this average response. The position of the point of inflection of the curve seemed to be a function of time

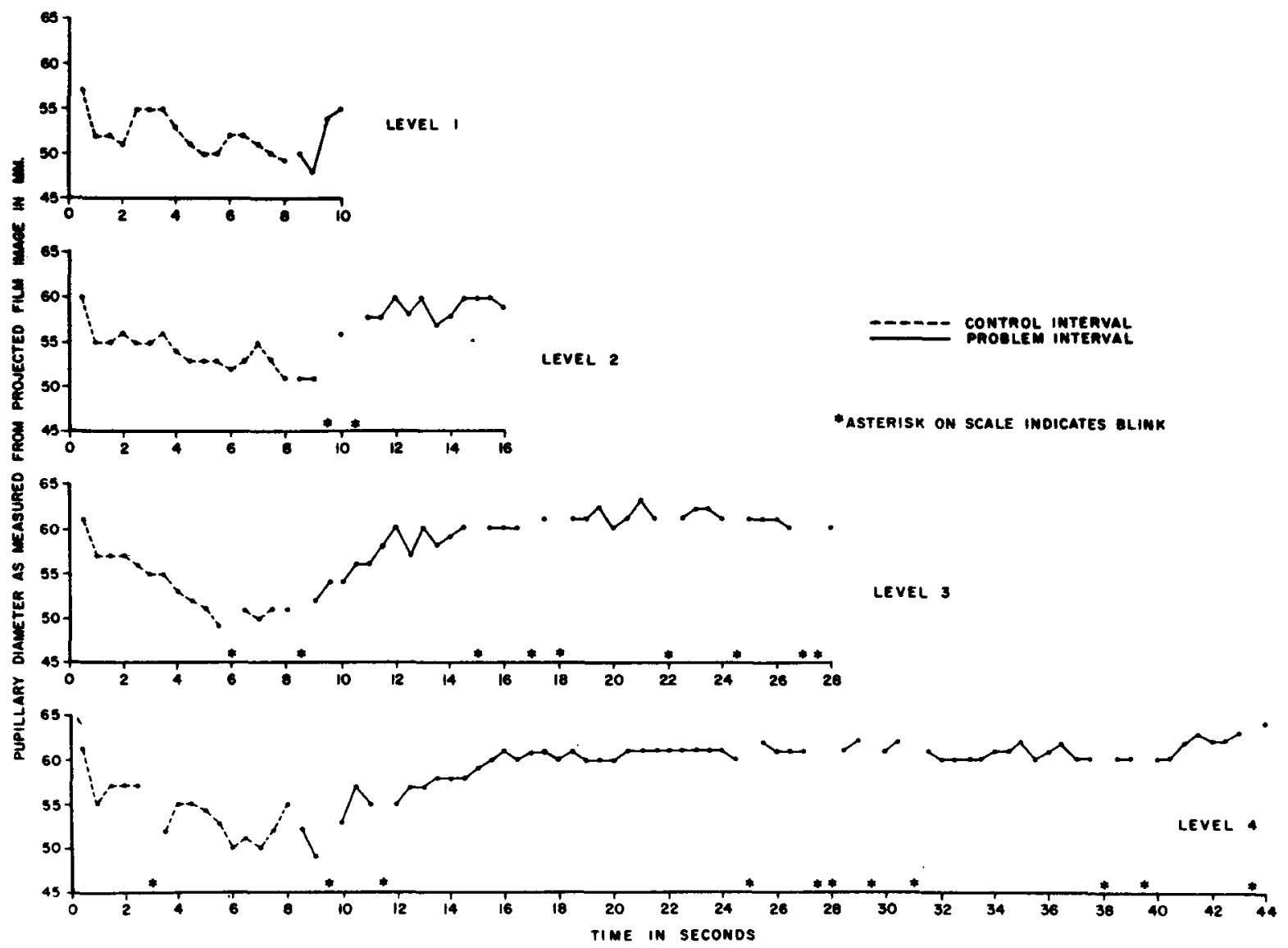

Fig. 4. Pupillary response curves of a typical $S$ for one item from each level of difficulty. 
after item presentation. Since responses to Level 1 items terminated (i.e., a solution was offered) before the inflection point of the average curve was reached, it is reasonable to explain the difference in the amount of dilation as a function of the length of the interval during which processing occurred rather than the type of processing occurring.

\section{Measurement of Percentage of Pupillary Dilation}

The pupillary response was measured as percentage of dilation in this study as suggested by Hess and Polt (1964). The two measurement procedures differed. In the present study, all data points within the control and problem solving intervals were averaged rather than averaging only points within the $2.5 \mathrm{sec}$ intervals preceding and following presentation of the stimulus. Problems associated with both of these procedures are discussed, and the suitability of a percentage measure of dilation is questioned, in Payne, Parry and Harasymiw (1967). They conclude that simple difference measures may be preferable to percentage measures.

\section{Conclusions}

The results of this study and others indicate that the pupillary dilation reflex is a sensitive and apparently reliable indicator of internal information processing. The fact that this response is related to activity in the sympathetic nervous system makes it a signal that should be better understood. However, the relation between activity in the sympathetic nervous system (or, more narrowly, thinking processes) and pupillary responses is only beginning to be studied and much research remains to be done to unravel these relationships.

\section{REFERENCES}

HESS, E. H., \& POLT, J. M. Pupil size in relation to mental activity during simple problem-solving. Science, 1964, 143, 1190-1192.

KAHNEMAN, D., \& BEATTY, J. Pupil diameter and load on memory. Science, 1966, 154, 1583-1585.

KAHNEMAN, D., \& BEATTY, J. Pupillary responses in a pitchdiscrimination task. Percept. \& Psychophys, 1967, 2, $101-105$.

PAYNE, D. T., PARRY, M. E., \& HARASYMIW, S. J. A comparison of percentage of pupillary dilation with other measures of difficulty of mental multiplication items. Research Bulletin 67-42. Princeton, N.J.: Educational Testing Service, 1967.

WINER, B. J. Statistical principles in experimental design. New York: McGraw-Hill, 1962.

WOODMANSEE, J. J. Methodological problems in pupillographic experiments. Paper presented at the American Psychological Association, New York, September, 1966.

NOTES

1. The authors wish to thank Dr. Felix F. Kopstein and Dr. Donald M. Medley for their very helpful ideas and suggestions on this project.

2. Address: Educational Testing Service, Princeton, N.J. 08540.

3. The kind of operation that varies among items if digit values are not controlled is the "carry" operation. For the digit values specified every product is a two-digit number which implies that an equal number of "carry" operations is involved for all items at each level.

4. The average values analyzed in the analysis of variance of the pupillary dilation are computed from samples of widely varying size. The reported analysis is therefore statistically inappropriate. The authors have been unsuccessful at completing a more appropriate design and therefore report the above tentative results. The reader is cautioned to interpret the results with reservations.

(Accepted for publication May 7, 1968.) 